\title{
Perturbations in imprinted methylation from assisted reproductive technologies but not advanced maternal age in mouse preimplantation embryos
}

Audrey J. Kindsfather ${ }^{1,2}$, Megan A. Czekalski ${ }^{1,2}$, Catherine A. Pressimone ${ }^{1,2}$, Margaret P. Erisman ${ }^{1,2}$ and Mellissa R. W. Mann ${ }^{1,2^{*}}$ (i)

\begin{abstract}
Background: Over the last several decades, the average age of first-time mothers has risen steadily. With increasing maternal age comes a decrease in fertility, which in turn has led to an increase in the use of assisted reproductive technologies by these women. Assisted reproductive technologies (ARTs), including superovulation and embryo culture, have been shown separately to alter imprinted DNA methylation maintenance in blastocysts. However, there has been little investigation on the effects of advanced maternal age, with or without ARTs, on genomic imprinting. We hypothesized that ARTs and advanced maternal age, separately and together, alter imprinted methylation in mouse preimplantation embryos. For this study, we examined imprinted methylation at three genes, Snrpn, Kcnq10t1, and H19, which in humans are linked to ART-associated methylation errors that lead to imprinting disorders.
\end{abstract}

Results: Our data showed that imprinted methylation acquisition in oocytes was unaffected by increasing maternal age. Furthermore, imprinted methylation was normally acquired when advanced maternal age was combined with superovulation. Analysis of blastocyst-stage embryos revealed that imprinted methylation maintenance was also not affected by increasing maternal age. In a comparison of ARTs, we observed that the frequency of blastocysts with imprinted methylation loss was similar between the superovulation only and the embryo culture only groups, while the combination of superovulation and embryo culture resulted in a higher frequency of mouse blastocysts with maternal imprinted methylation perturbations than superovulation alone. Finally, the combination of increasing maternal age with ARTs had no additional effect on the frequency of imprinted methylation errors.

Conclusion: Collectively, increasing maternal age with or without superovulation had no effect of imprinted methylation acquisition at Snrpn, Kcnq10t1, and H19 in oocytes. Furthermore, during preimplantation development, while ARTs generated perturbations in imprinted methylation maintenance in blastocysts, advanced maternal age did not increase the burden of imprinted methylation errors at Snrpn, Kcnq10t1, and H19 when combined with ARTs. These results provide cautious optimism that advanced maternal age is not a contributing factor to imprinted methylation errors in embryos produced in the clinic. Furthermore, our data on the effects of ARTs strengthen the need to advance clinical methods to reduce imprinted methylation errors in in vitro-produced embryos.

Keywords: Genomic imprinting, DNA methylation, Fertility, Assisted reproductive technologies, Maternal age, Mouse

\footnotetext{
* Correspondence: mannmr@mwri.magee.edu

${ }^{1}$ Department of Obstetrics, Gynecology and Reproductive Sciences,

University of Pittsburgh School of Medicine, 204 Craft Ave, Pittsburgh, PA

15213, USA

${ }^{2}$ Magee-Womens Research Institute, 204 Craft Ave, Pittsburgh, PA 15213, USA
}

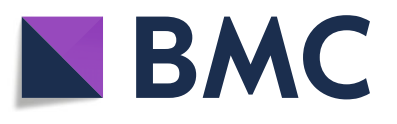

(c) The Author(s). 2019 Open Access This article is distributed under the terms of the Creative Commons Attribution 4.0 International License (http://creativecommons.org/licenses/by/4.0/), which permits unrestricted use, distribution, and reproduction in any medium, provided you give appropriate credit to the original author(s) and the source, provide a link to the Creative Commons license, and indicate if changes were made. The Creative Commons Public Domain Dedication waiver (http://creativecommons.org/publicdomain/zero/1.0/) applies to the data made available in this article, unless otherwise stated. 


\section{Introduction}

Recent societal trends have resulted in women delaying pregnancy until later in their childbearing years. As such, over the last several decades, maternal age at the first and subsequent pregnancies has risen steadily [1]. Notably, birth rates have significantly increased among women $35-55$ years of age, which is considered advanced maternal age, labeling the current decade as an "epidemic of age-related infertility" [1-3]. This increase in maternal age has contributed in part to the tripling of infertility rates since 1984 ( 5\% to $15 \%)[4,5]$. After the age of 35, a woman's fertility declines, and in the likelihood that a pregnancy is achieved, the risk of adverse outcomes increases $[6,7]$. Thus, it is crucial to understand the molecular consequences of advanced maternal age in gametes and embryos.

One effect of aging is nuclear changes in DNA methylation. A landmark paper in 2005 discovered that as monozygotic twins age, their DNA methylomes become increasingly different [8]. Subsequent studies found that age-related changes in DNA methylation accumulate over a lifetime [9-11]. In females, reproductive or oocyte aging is defined as a progressive decline in oocyte numbers and quality $[12,13]$. To date, only a couple of studies have investigated DNA methylation or the catalytic enzymes involved, DNA methyltransferases (DNMTs), in aged oocytes or the resulting embryos. Advanced maternal age leads to alterations in gene expression in oocytes, including Dnmt1 and Dnmt3b, as well as a decrease in DNMT1, DNMT3A, and DNMT3B protein levels $[14,15]$. Furthermore, these oocytes produced embryos with a decrease in global DNA methylation during preimplantation embryo development [14].

Genomic imprinting is another epigenetic mechanism that employs DNA methylation. Here, one copy of a gene is silenced based on the parental origin of inheritance, while the other parental allele is expressed [16]. These sex-specific DNA methylation marks are acquired during gametogenesis and maintained during preimplantation development $[17,18]$. To date, the effects of advanced maternal age on the acquisition and maintenance of imprinted DNA methylation in oocytes and resulting preimplantation embryos, respectively, have not been investigated.

In response to the age-related fertility decline, women of advanced maternal age frequently turn to ARTs as medical treatments for subfertility, which often represent the best recourse for achieving a pregnancy. While generally considered safe, ARTs, including hormone-induced ovarian hyperstimulation (superovulation) and in vitro embryo culture, have been linked to imprinting disorders. Significantly, there are more cases of Beckwith-Wiedemann syndrome (BWS), Silver-Russell syndrome (SRS), Angelman syndrome (AS), and Prader-Willi syndrome (PWS) due to imprinted methylation errors in children conceived with ARTs compared to the general population, suggesting that imprinting defects are exacerbated by ARTs [19-35]. This is supported by our published findings, which have demonstrated that ARTs lead to imprinted methylation errors in early mouse and likely human embryos from young mothers [36-42], although imprinted methylation acquisition in mouse oocytes is not altered by superovulation [43]. However, there has been little investigation on the effects of advanced maternal age in combination with ARTs on genomic imprinting.

In this study, we hypothesized that advanced maternal age leads to perturbations in imprinted methylation acquisition in oocytes and/or imprinted methylation maintenance in blastocysts. To facilitate this analysis, as well as relate our findings to maternal age in humans, we produced a model of maternal aging, extending from puberty to reproductive senescence, that more precisely relates human and mouse ages $[44,45]$. Since imprinted gene regulation is well conserved between mouse and human [18, 46], and imprinting disorders in ART-conceived children are associated with methylation perturbations at SNRPN, KCNQ1OT1, and H19 [19-35], we investigated imprinted methylation at Snrpn, Kcnq1ot1, and H19 in our mouse model. We also hypothesize that a combination of advanced maternal age with ARTs results in a higher frequency of blastocysts with a loss of imprinted methylation than any ART alone. Prior to addressing this hypothesis, we first characterized the effects of various ARTs on imprinted methylation maintenance in mouse blastocysts. More specifically, we examined superovulation and embryo culture, singly and together, and with respect to the latter, we also investigated low and ambient oxygen conditions, since low oxygen has been recommended based on improved embryo development [47-50]. We found that imprinted methylation acquisition and maintenance were unaffected by increasing maternal age, and that the frequency of imprinted methylation errors in blastocysts was unchanged when increasing maternal age was combined with ARTs. In conclusion, while a combination of ARTs altered imprinted methylation at Snrpn, Kcnq1ot1, and H19 in mouse blastocysts, advanced maternal age did not increase the burden of imprinted methylation errors when combined with ARTs.

\section{Results}

Imprinted methylation acquisition in oocytes was unaltered by advanced maternal age

During gamete development, extensive genome-scale epigenetic transitions occur, including erasure of DNA methylation in primordial germ cells and subsequent acquisition of sex-specific imprinted methylation marks $[17,18]$. Given that studies showed that advanced maternal age leads to changes in gene expression and protein 
levels of DNA methyltransferases [14, 15], the first hypothesis we tested was whether advanced maternal age, without ARTs, altered the ability of oocytes to normally acquire imprinted methylation marks. To address this, germinal vesicle (GV) oocytes from spontaneously ovulating female mice in four maternal age groups were examined for their imprinted methylation status at the Snrpn, Kcnq1ot1, and H19 imprinting control regions (ICRs), which are associated with AS, PWS, BWS, and SRS. Any perturbation in the acquisition of imprinted methylation would be apparent by this late preovulatory stage of oogenesis [51]. Female mice were categorized based on a model we produced for maternal aging that more precisely relates human and mouse ages [44, 45]. Young maternal age in mice of $>2-6$ months corresponded to humans of $15-25$ years. Middle maternal age mice of > 6-10 months paralleled > 25-35 years in humans, while advanced maternal age of $>10-14$ months in mice corresponded to > 35-45 years in humans (Additional file 1: Figure S1). Female mice in these three age groups were retired breeders with proven fertility. Virgin females between 1.5 to 2 months old served as controls. During oocyte growth, Snrpn and Kcnq1ot1 acquire DNA methylation and by the germinal-vesicle and mature MII stages, this acquisition is complete [69, 71, 73-75]. By comparison, H19 remains unmethylated in oocytes. Our analysis showed that, except for one oocyte from a young female with $40 \%$ Kcnq1ot1 methylation, GV oocytes from all maternal age groups had the same high levels of Snrpn and Kcnq1ot1 methylation and the same low levels of H19 methylation as GV oocytes from virgin females (Fig. 1). These results indicate that imprinted methylation was acquired normally during oogenesis in females of advanced maternal age.

\section{Imprinted methylation acquisition was unchanged by advanced maternal age combined with superovulation}

We previously showed that superovulation (SO) does not affect imprinted methylation acquisition as assessed in meiosis II (MII) oocytes from virgin females [43]. However, whether the combination of maternal age and $\mathrm{SO}$ impacts acquisition of imprinted methylation has not been investigated. To address this, MII oocytes from superovulated virgin (1.5-2 months old) and advanced maternal age ( $>10$ months old) females were assessed for imprinted methylation at the Snrpn, Kcnq1ot1, and H19 ICRs. Compared to controls, MII oocytes from advanced-aged females displayed similar hypermethylation of the Snrpn and Kcnq1ot1 ICRs and similar hypomethylation of the H19 ICR (Fig. 2). These data indicate that imprinted methylation acquisition was not adversely affected by advanced maternal age plus $\mathrm{SO}$, and are consistent with our previous published data on $\mathrm{SO}$ alone [43].

\section{Postzygotic imprinted methylation maintenance was} unaltered by advanced maternal age

Another important stage of global epigenetic alterations is preimplantation development, when genome-scale demethylation occurs $[17,18]$. However, some genomic regions are protected from the demethylation machinery. These regions include ICRs, which maintain their allelespecific methylation patterns during this period. In previous studies, we found that while SO did not alter imprinted methylation acquisition in oocytes [43], it led to perturbations in imprinted methylation maintenance in preimplantation embryos [40]. Here, we tested the hypothesis that advanced maternal age leads to postzygotic changes in imprinted methylation during preimplantation development. Thus, blastocysts from spontaneously ovulating virgin, young, middle-, and advanced-aged females were assessed for Snrpn, Kcnq1ot1, and H19 imprinted methylation. Similar to controls, blastocysts from females of increasing maternal age had no significant difference in imprinted methylation, i.e., the maternal Snrpn, maternal Kcnq1ot1, and paternal H19 ICRs possessed normal hypermethylation ( $\geq 75 \%$ methylation), while the paternal Snrpn, paternal Kcnq1ot1, and maternal H19 ICRs harbored normal hypomethylation $(<20 \%$ methylation) (Fig. 3; Additional file 1: Figures S2, S3, and S4). These results indicate that maintenance of imprinted methylation was not adversely affected by advanced maternal age.

As females age, they are naturally prone to weight gain [52]. Several studies in humans and mice have reported that maternal weight and nutrition prior to conception and during pregnancy alters DNA methylation in their offspring [53-55]. Thus, we questioned whether there was a relationship between maternal weight and imprinted methylation in blastocyst-stage embryos described above. While we observed a positive correlation between maternal age and weight, no association was found between maternal weight and imprinted methylation levels at the Snrpn, Kcnq1ot1, and H19 ICRs in blastocysts (Additional file 1: Figure S5). This suggests that natural age-associated weight gain per se does not perturb maintenance of imprinted methylation in preimplantation embryos.

\section{Additive effects of ARTs on imprinted methylation errors in blastocysts}

In a previous study, we reported that a combination of $\mathrm{SO}$ and embryo culture (EC) increased the frequency of embryos with a loss of $H 19$ imprinted expression when compared to embryos that were in vivo-derived or derived from SO alone [39]. A comprehensive study examining imprinted methylation comparing maternal age with and without ARTs has not previously been performed. Thus, prior to investigating the effects of maternal age with ARTs on imprinted methylation, we first characterized the effects of ARTs (SO only, EC only, and 


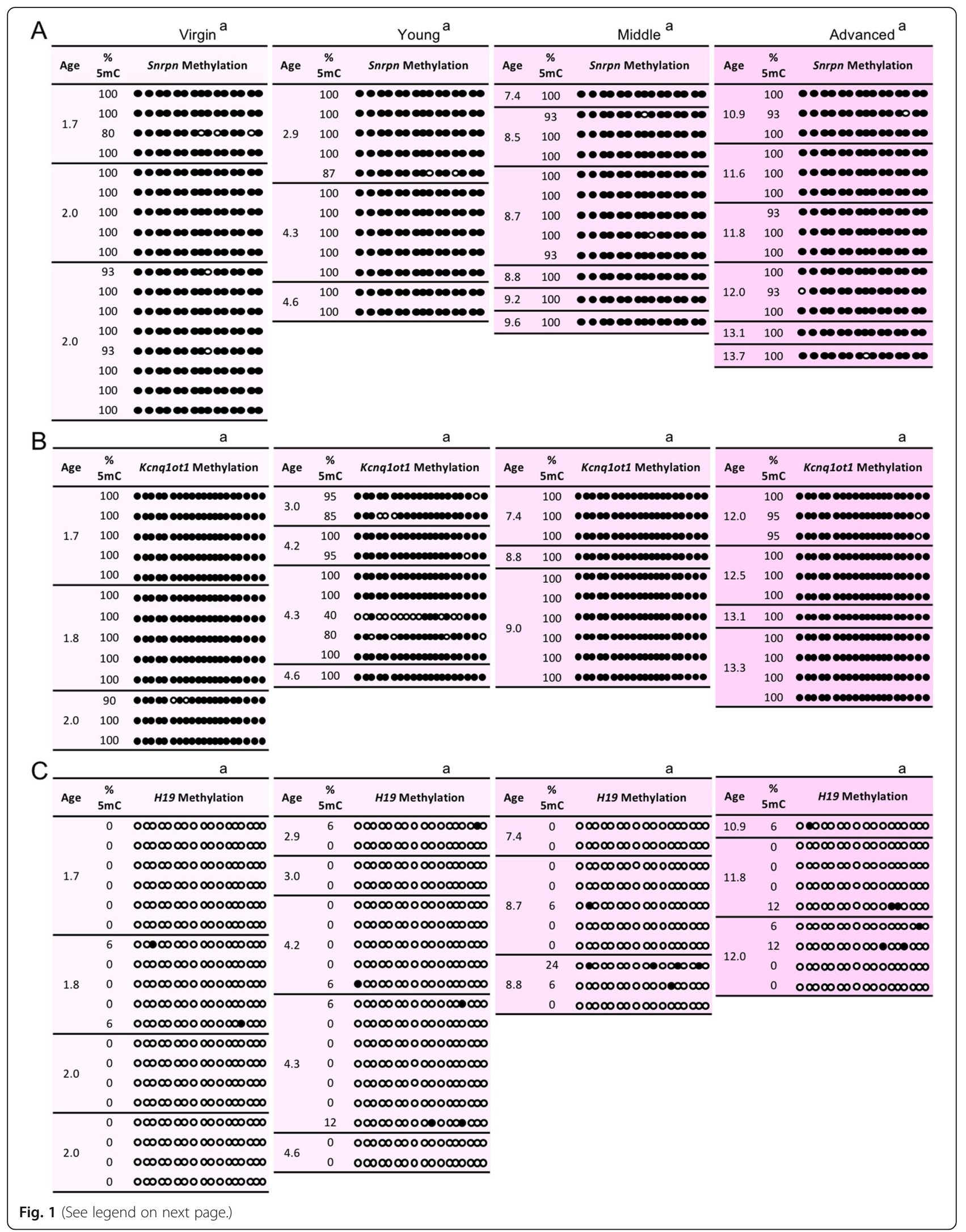


(See figure on previous page.)

Fig. 1 Imprinted methylation acquisition at the (a) Snrpn, (b) Kcnq1ot1, and (c) H19 ICRs in germinal vesicle oocytes was unaltered by increasing maternal age. Each box shows oocytes from spontaneously ovulating females with maternal age in months (mths) $(n=3-4$ females per age group). Each line of circles represents the CpGs in the Snrpn, Kcnq10t1, or H19 ICRs in an individual oocyte ( $n=13-19$ oocytes per age group). Oocytes were excluded if sequenced DNA clones ( $n=5-8 /$ denuded oocyte) exhibited more than one methylation pattern, suggestive of cumulus cell contamination. Black circles, methylated CpGs; white circles, unmethylated CpGs; \%5mC, percentage of 5-methyl CpGs over the total number of CpGs for a specific ICR. Same letter (a), no statistically significant difference between groups

both $\mathrm{SO}+\mathrm{EC}$ ) on imprinted methylation maintenance in mouse blastocysts compared to no ARTs (spontaneous ovulation, in vivo derived) using virgin females. Compared to the no ARTs group, we observed a significant loss in imprinted methylation in blastocysts from the SO group, the EC group, as well as the $\mathrm{SO}+\mathrm{EC}$ group at the maternal Snrpn, maternal Kcnq1ot1, and the paternal H19 ICRs (Fig. 4; Additional file 1: Figures S6, S7, and S8; Additional file 1: Table S1).

A comparison between ART groups revealed no significant difference between the SO only and EC only groups for the frequency of blastocysts with imprinted methylation loss at the maternal Snrpn, maternal Kcnq1ot1, and paternal H19 ICRs (Fig. 4; Additional file 1: Figures S6, S7, and S8; Additional file 1: Table S1). However, compared to $\mathrm{SO}$ alone, a significant increase in the frequency of imprinted methylation loss occurred at the maternal Snrpn and maternal Kcnq1ot1 ICRs in blastocysts from the SO+EC groups, but not for the paternal H19 ICR. With regard to EC, no significant difference was found between the $\mathrm{EC}$ only and $\mathrm{SO}+\mathrm{EC}$ groups for the three imprinted genes.

Embryo culture under low oxygen conditions has been recommended since numerous studies utilizing low oxygen have reported improved embryo development [47-50]. However, few studies have examined the effects of low and high oxygen concentrations on imprinted methylation. Here, we compared the effects of SO+EC using low and high $\mathrm{O}_{2}$ concentrations $\left[5 \% \mathrm{O}_{2}\right.$ and $~ 21 \%$ $\mathrm{O}_{2}$ in ambient air, respectively] on imprinted methylation maintenance. No significant difference was found in the frequency of embryos with Snrpn, Kcnq1ot1, and H19 methylation loss in SO+EC groups whether cultured in 5\% or $\sim 21 \% \mathrm{O}_{2}$ (Fig. 4; Additional file 1: Figures S6, S7, and S8; Additional file 1: Table S1). These results are consistent with those reported by de Waal et al. [56] and Ghosh et al. [57], who reported no difference in DNA methylation perturbations in mouse and human placentas between the two oxygen tensions, although both differed from controls.

For all treatment comparisons, no significant change in methylation of the normally unmethylated allele was found for any gene. Overall, these results indicate that the frequency of imprinted methylation perturbations was similar between SO and EC, while for ICRs harboring maternal methylation, $\mathrm{SO}+\mathrm{EC}$ produces an additive effect with a greater frequency of blastocysts with perturbations in imprinted methylation than $\mathrm{SO}$ alone.
One question that arises from these experiments is whether all females undergoing ARTs produce embryos with imprinted methylation errors, or whether some females have embryos that maintain imprinted methylation while other females generate embryos that lose imprinted methylation. To address this question, blastocysts that were analyzed above according to ART group were also evaluated by individual litters within a treatment group. Compared to controls, every litter had at least one blastocyst that lost imprinted methylation in the SO only, EC only, and SO+EC groups with the frequency of imprinted methylation errors reflective of the conditions (Additional file 1: Figure S9). These data indicate that it is exclusively ARTs acting at the individual embryo level, rather than a female or batch effect, which contributes to imprinted methylation errors.

\section{No additional burden of imprinted methylation maintenance errors with a combination of advanced maternal age and ARTs}

Finally, we tested the hypothesis that a combination of advanced maternal age and ARTs would result in a higher frequency of blastocysts with imprinted methylation errors than ARTs alone. To test this hypothesis, we compared embryos in the virgin and advanced age no ARTs groups with those in the virgin and advanced age $\mathrm{SO}+\mathrm{EC}$ groups. A significant loss in imprinted methylation occurred in blastocysts from the advanced age $\mathrm{SO}+\mathrm{EC}$ group compared to advanced age no ARTs group, similar to these groups from virgin females (Fig. 5; Additional file 1: Figures S10, S11, and S12; Additional file 1: Table S2). However, the comparison between virgin $\mathrm{SO}+\mathrm{EC}$ and advanced maternal age plus $\mathrm{SO}+\mathrm{EC}$ revealed no significant change in the frequency of blastocysts with imprinted methylation perturbations at the maternal Snrpn, maternal Kcnq1ot1, and paternal H19 ICRs. These data indicate that advanced maternal age does not further increase the burden of imprinted methylation errors when combined with ARTs.

\section{Discussion}

As women increasingly delay childbirth, it is important to understand the effects of reproductive aging on oocyte and embryo quality so that women can be informed of the potential risks to both the pregnancy and the future health of the baby. As fertility declines with 


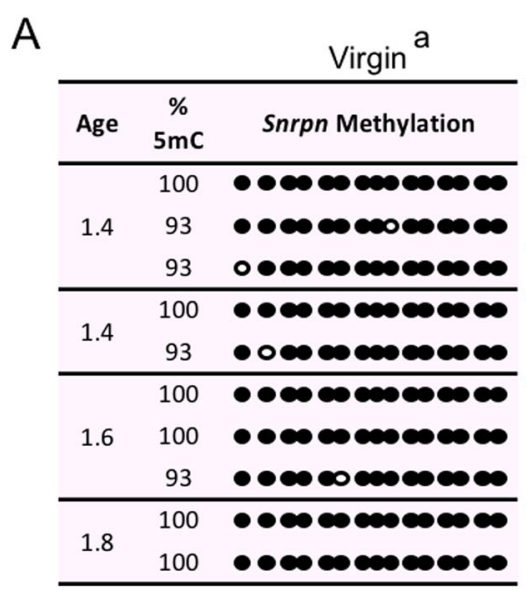

\begin{tabular}{|c|c|c|}
\hline Age & $\begin{array}{c}\% \\
5 \mathrm{mC}\end{array}$ & Snrpn Methylation \\
\hline 11.1 & $\begin{array}{l}100 \\
100\end{array}$ & 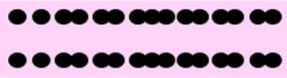 \\
\hline 12.4 & 100 & 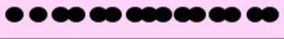 \\
\hline 12.4 & $\begin{array}{l}100 \\
100 \\
\end{array}$ & 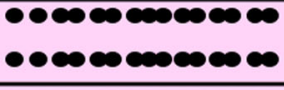 \\
\hline 12.6 & $\begin{array}{l}100 \\
100 \\
100 \\
\end{array}$ & 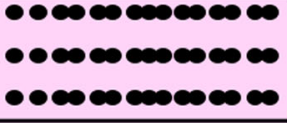 \\
\hline 12.8 & $\begin{array}{l}100 \\
100 \\
100\end{array}$ & 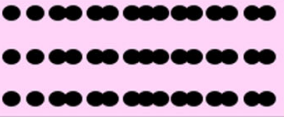 \\
\hline
\end{tabular}

B

\begin{tabular}{|c|c|c|}
\hline Age & $\begin{array}{c}\% \\
5 \mathrm{mC}\end{array}$ & Kcnq1ot1 Methylation \\
\hline 1.4 & 100 & -ம⿻ \\
\hline \multirow{3}{*}{1.4} & 100 & ocoese \\
\hline & 100 & \\
\hline & 100 & oper \\
\hline \multirow{4}{*}{1.6} & 100 & 0000 \\
\hline & 100 & 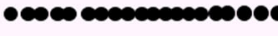 \\
\hline & 100 & \\
\hline & 100 & oper \\
\hline \multirow{2}{*}{1.8} & 95 & 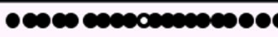 \\
\hline & 100 & - coco eseser \\
\hline
\end{tabular}

\begin{tabular}{|c|c|c|}
\hline Age & $\begin{array}{c}\% \\
5 \mathrm{mC}\end{array}$ & Kcnq1ot1 Methylation \\
\hline \multirow{2}{*}{11.1} & 100 & - cosece \\
\hline & 100 & - \\
\hline \multirow{2}{*}{12.0} & 95 & - coesen \\
\hline & 100 & ๑ம⿻上丨 \\
\hline \multirow[t]{2}{*}{12.4} & 100 & - coescese \\
\hline & 100 & - \\
\hline \multirow[t]{2}{*}{12.4} & 100 & $\bullet$ \\
\hline & 100 & ๑юces \\
\hline \multirow{2}{*}{12.6} & 100 & - cescencescen \\
\hline & 100 & ovesen \\
\hline \multirow{3}{*}{12.8} & 90 & - \\
\hline & 100 & \\
\hline & 100 & - \\
\hline
\end{tabular}

C

\begin{tabular}{|c|c|c|}
\hline Age & $\begin{array}{c}\% \\
5 \mathrm{mC}\end{array}$ & Kcnq1ot1 Methylation \\
\hline \multirow{2}{*}{1.4} & 0 & $, \infty \infty \infty, \infty \infty \infty \infty \infty$ \\
\hline & 0 & $0 \infty \infty \infty \infty, \infty, 000 \infty$ \\
\hline \multirow{2}{*}{1.4} & 6 &.$<\infty \infty 000000000$ \\
\hline & 0 & $0 \infty \infty \infty, \infty, \infty \infty \infty$ \\
\hline \multirow{2}{*}{1.4} & 0 & $0 \infty \infty \infty 000000000$ \\
\hline & 0 & $0 \infty \infty \infty, \infty, 0000 \infty$ \\
\hline \multirow{4}{*}{1.6} & 6 & $0 \infty \infty \infty, \infty) \infty \infty$ \\
\hline & 0 & , $\infty \infty \infty, \infty \infty \infty \infty \infty$ \\
\hline & 0 & $0 \infty \infty \infty, \infty \infty \infty \infty \infty$ \\
\hline & 6 & $\bullet \infty \infty \infty, \infty \infty \infty \infty \infty$ \\
\hline
\end{tabular}

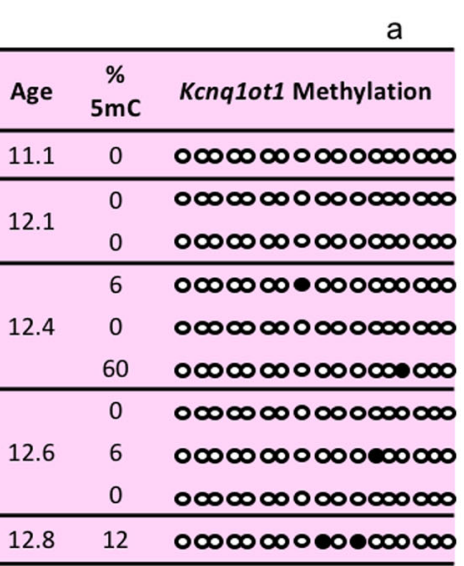

Fig. 2 Imprinted methylation acquisition at the (a) Snrpn, (b) Kcnq1ot1, and (c) H19 ICRs in MII oocytes was unchanged by increasing maternal age combined with superovulation. Each box shows oocytes from superovulated females with maternal age in months (mths) ( $n=3-4$ females per age group; $n=10-13$ oocytes per age group). See Fig. 1 for details. Same letter (a), no statistically significant difference between groups

increasing maternal age, many women turn to fertility treatments to conceive [3], further contributing to the environmental stressors on the embryo. However, to date, there has been little investigation on the effects of advanced maternal age, with or without ARTs, on genomic imprinting. Overall, our data showed that while 

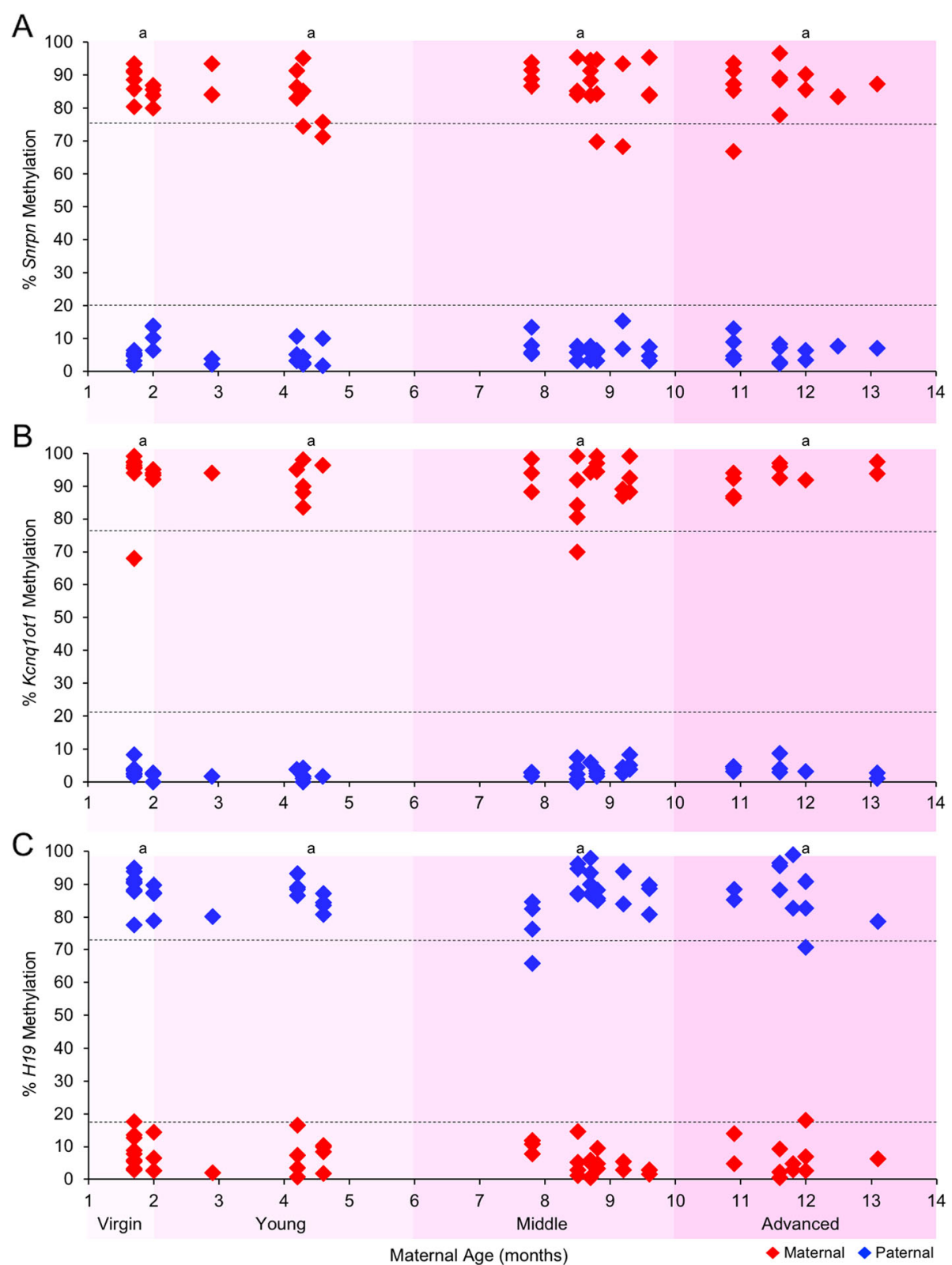

Fig. 3 Advanced maternal age did not perturb imprinted methylation maintenance of the (a) Snrpn, (b) Kcnq1ot1, and (c) H19 ICRs in blastocysts. Embryos were derived from spontaneously ovulating females. Diamonds represent the mean methylation levels of maternal (red) or paternal (blue) alleles for the Snrpn, Kcnq10t1, and H19 ICRs in individual blastocysts ( $n=9-22$ embryos, $n=3-7$ females per age group). Same letter (a), no statistically significant difference between groups

ARTs alter imprinted methylation maintenance of Snrpn, Kcnq1ot1, and H19 in preimplantation embryos, advanced maternal age had no effect on imprinted methylation acquisition in oocytes or on imprinted methylation maintenance in blastocysts. Moreover, advanced maternal age did not further increase the burden of imprinted methylation errors at Snrpn, Kcnq1ot1, and H19 when combined with ARTs. These results support cautious optimism that advanced maternal age is not a contributing factor to imprinted methylation errors in embryos produced in the clinic. Furthermore, our data on the effects of ARTs strengthen the need to advance clinical methods to reduce imprinted methylation errors in in vitro-produced embryos.

The results of our study are consistent with those reported by others. A comparison of young (2-2.5 months) and advanced aged ( 10-11 months) females failed to detect any change in imprinted methylation in E10.5 embryos, although there was greater embryo demise [58]. Our analysis of imprinted methylation in blastocysts from 


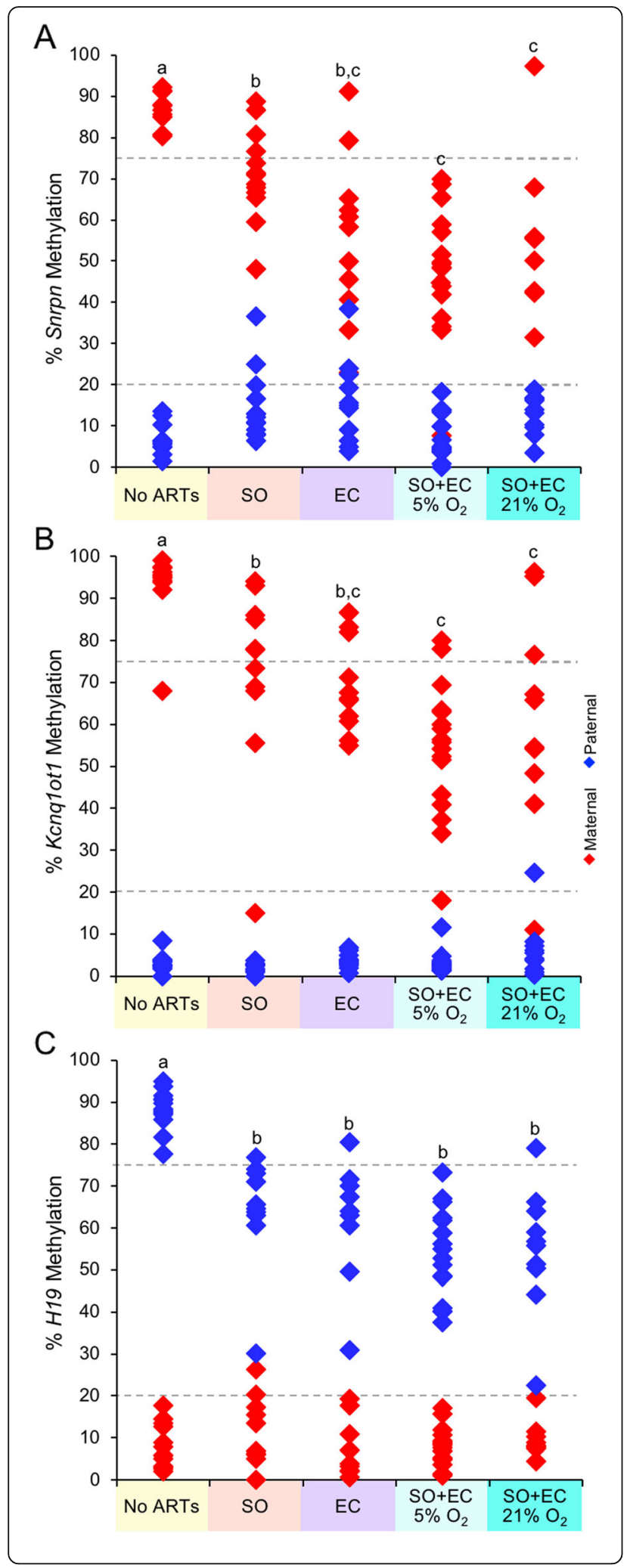

Fig. 4 ARTs generated a loss of imprinted methylation maintenance at the (a) Snrpn, (b) Kcna1ot1, and (c) H19 ICRs in blastocysts from young mothers. Diamonds represent the mean methylation levels of maternal (red) or paternal (blue) alleles for Snrpn, Kcnq1ot1, and H19 ICRs in individual embryos ( $n=9-17$ blastocysts; $n=3-4$ females per treatment group). SO, superovulation; EC, embryo culture. Letters $(a, b, c)$, a statistically significant difference between groups (Additional file 1: Table S1)

females of increasing age suggests that this embryo wastage was not due to imprinted methylation maintenance errors arising during preimplantation development. In another study, DNA methylation was unchanged at imprinted genes in E16.5 embryos from advanced aged (15 months old) females compared to young ( 1 month old) mothers, although an increase in DNA methylation at imprinted genes was reported for E16.5 placentas from advanced aged mothers (15 months old) [59]. It should be noted only 1-2 CpG sites were assessed in this analysis for total methylation, so it is unclear which parental allele may have gained methylation. Finally, in another report that investigated the epidemiological association in Japan between imprinting disorders, ARTs and advanced maternal age found that ART-conceived children with PWS were more likely to have Snrpn methylation errors than spontaneously conceived children with PWS when their mothers were less than 37 years old [35]. However, when mothers were more than 38 years old, there was no difference in the frequency of PWS caused by DNA methylation errors in spontaneously versus ARTconceived children. Instead, a significantly higher risk of maternal uniparental disomy occurred in ART-conceived children of these mothers, suggesting that the etiology of imprinting disorders in children of women of advanced maternal age was due to chromosomal segregation errors in aged oocytes. Thus, the effect of ARTs on imprinted DNA methylation was independent of maternal age, which supports our conclusions. Overall, the evidence that advanced maternal age did not further increase the burden of imprinted methylation errors when combined with ARTs provides important information for women over the age of 35 .

Numerous ARTs are employed for in vitro production of mouse and human embryos. A complete clinical cycle may include ovarian stimulation, oocyte retrieval, in vitro fertilization or intracytoplasmic injection, embryo culture, oocyte or embryo cryopreservation, and embryo transfer to mothers. Several studies on the effects of multiple ARTs reported that combining ARTs increased the chance of imprinted methylation and expression errors when compared to single interventions [39, 40, 60-62]. Here, we found that $\mathrm{SO}+\mathrm{EC}$ produced an additive effect with a greater frequency of blastocysts with perturbations in maternal Snrpn and Kcnq1ot1 imprinted methylation 


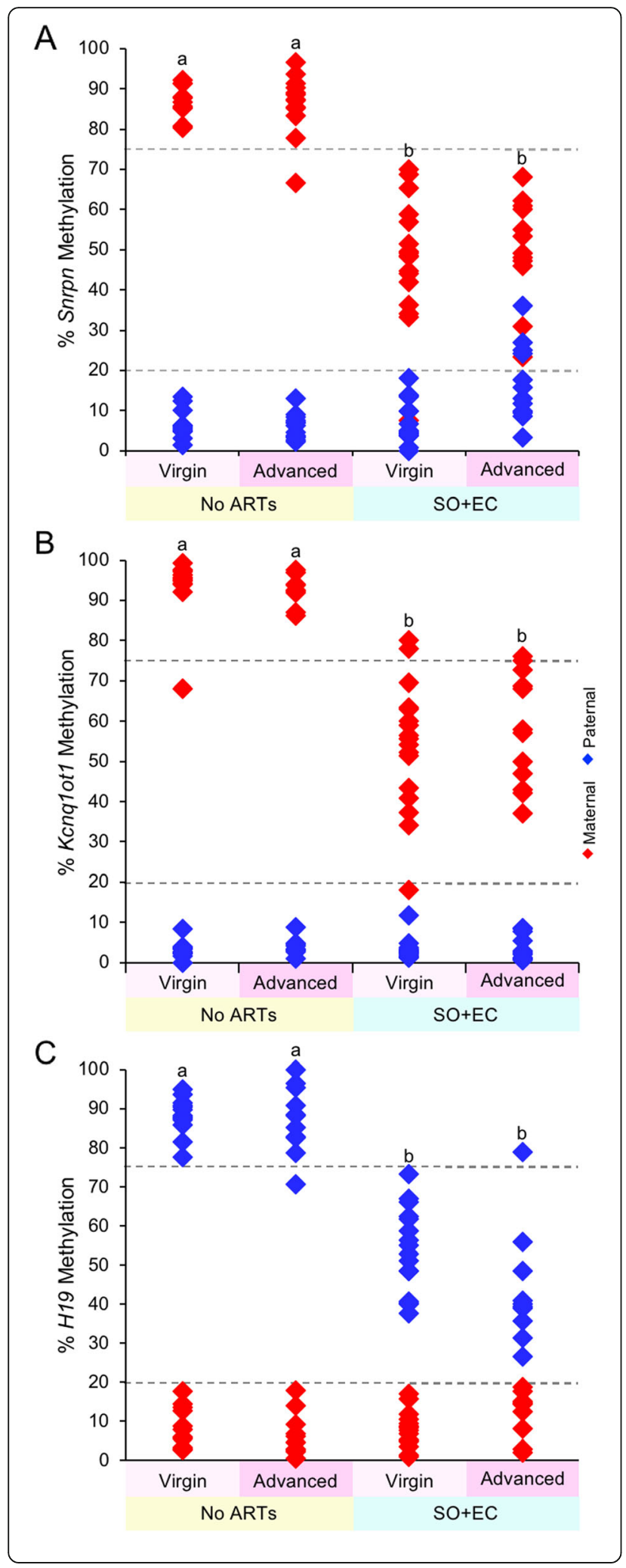

Fig. 5 Increasing maternal age plus ARTs did not further increase imprinted methylation errors at the (a) Snrpn, (b) Kcnq1ot1, and (c) H19 ICRs in blastocysts. Diamonds represent the mean methylation levels of maternal (red) or paternal (blue) alleles for Snrpn, Kcna1ot1, and H19 ICRs in individual embryos ( $n=10-17$ blastocysts; $n=2-5$ females per treatment group). Letters ( $a, b, c)$, a statistically significant difference between groups (Additional file 1: Table S2)

than $\mathrm{SO}$ alone. These results suggest that $\mathrm{SO}$ and $\mathrm{EC}$ have independent effects on genes harboring maternal methylation. In contrast, there was no significant difference in the frequency of blastocysts with H19 imprinted methylation perturbations between the SO only, EC only, and SO+EC groups. This may relate to the fact that $H 19$ possesses paternal, sperm-inherited methylation, and suggests that $\mathrm{SO}$ and $\mathrm{EC}$ also converge on the same molecular pathway during preimplantation development. Future studies are needed to investigate the molecular mechanisms regulating imprinted methylation maintenance and how they may be perturbed by ARTs.

While the focus of this study was centered on imprinted methylation, the possibility exists that advanced maternal age may impair DNA methylation or histone modifications at other regions of the genome. In one study, global methylation that was assessed by immunofluorescence with 5$\mathrm{mC}$ antibody showed a slight but significant decrease in signal in preimplantation embryos from middle-aged mice (8-10 months) compared to young females (1.5-2 months) [14]. Three epigenome-wide association studies examining global DNA methylation in children of older mothers found individual CpG sites within different genomic regions that were significantly correlated with advanced maternal age [63-65]. However, Ghosh et al. [57] reported that while global and LINE1 methylation errors differed between control and ART placentas of young mothers $(<$ 35 years old), there was little difference in DNA methylation in older mothers ( $>35$ years old), pointing towards ARTs rather than maternal age as a contributing factor. In three studies that investigated histone modifications, histone 4 lysine 12 (H4K12) acetylation was improperly retained in MII oocytes from older mice and humans [66, 67], while histone methylation (H3K9me3, H3K36me2, H3K79me2, and H4K20me2) was abnormally decreased in GV and MII oocytes from advanced age (11 months old) compared to young (2 months old) mice [68]. Thus, future investigations are required to better understand the effects of advanced maternal age, with and without ARTs, on global DNA methylation and histone modifications.

A limitation of this study is the longevity of our animal model as compared to that of humans. While we found that female mice of advanced maternal age experienced a decreased ovarian reserve, producing fewer oocytes and embryos, the actual time of reproduction was 
maximally 14 months in our model. For humans, as a woman ages, so do her oocytes, with some oocytes reaching over 40 years before growth is initiated. The absolute time period for reproductive aging of 35-55 years could have significant impact on the ability to acquire and maintain imprinted methylation. Having said this, in our previous study on donated human preimplantation embryos, we found that the mean maternal age for embryos with normal methylation and abnormal levels at imprinted genes was 34 and 33 years old, which was not statistically different [42]. This suggests that advanced maternal age may not be an additional burden for imprinted methylation maintenance in humans, similar to the findings reported here for mice. To confirm this, future studies on donated human oocytes and preimplantation embryos from advanced aged women are needed to determine the impact of decades-long oocyte aging.

\section{Conclusion}

Increasing maternal age with or without superovulation had no effect of imprinted methylation acquisition in oocytes. While ARTs generated perturbations in imprinted methylation maintenance in blastocysts, advanced maternal age did not increase the burden of imprinted methylation errors when combined with ARTs. Overall, these results indicate that ARTs, and not maternal age, are the major contributor to imprinted methylation errors. Going forward, it is crucial to ascertain the molecular mechanisms underlying these imprinted methylation perturbations, as well as develop minimally invasive markers for identifying embryos with altered imprinted methylation. Knowledge gained from these studies will lead to advances that reduce the frequency of embryos with imprinting perturbations, and ultimately, ART-conceived children with imprinting disorders.

\section{Methods \\ Mice}

In this study, we utilized a mouse model suited for imprinting analyses, namely C57BL/6(CAST7) [B6(CAST7)] mice [69]. B6(CAST7) females had two copies of the Mus musculus castaneus chromosome 7 (CAST7) on a C57BL/ 6(B6) background, while B6 males (Jackson Laboratory) had two copies of the B6 chromosome 7; therefore, the offspring of these mice have one CAST7 chromosome 7 from the mother and one $\mathrm{B} 6$ chromosome 7 from the father. The mouse chromosome 7 contains numerous imprinted genes, including those investigated in this study: Snrpn, Kcnq1ot1, and H19. Multiple polymorphisms between the CAST7 and B6 alleles allow for the distinction of maternal versus paternal alleles, respectively, at imprinted loci on chromosome 7 in an embryo.

\section{Maternal aging model}

We produced a model for maternal aging, extending from puberty to reproductive senescence, that more precisely relates human and mouse ages [44, 45]. Female mice were then divided into four age groups (Additional file 1: Figure S1): virgin females between 1.5 to 2 months old that served as controls, young maternal age between $>2-6$ months old, middle maternal age between $>$ 6-10 months old, and advanced maternal age between $>10-14$ months of age. To control for possible subfertility, retired female breeder mice were used for the young, middle, and advanced age groups. These females at 2 months of age were set up with males as an active breeding pair and fertility was measured by the ability to continuously produce litters. Once they reach the targeted maternal ages, the breeding pair was dismantled, and the female was designated as a retired breeder with proven fertility. Each female was weighed to distill any effects of weight versus age. B6 male studs between 2 and 6 months of age were mated with control and experimental females. Matings were determined by the presence of a vaginal plug at 0.5 days post-coitum.

\section{Assisted reproductive technologies}

There were four treatment groups in this study: no ARTs, superovulation only, embryo culture only, and superovulation plus embryo culture. For the no ARTs group, germinal vesicle (GV) oocytes and blastocysts were collected from spontaneously ovulating females on embryonic day 3.5 (E3.5) in M2 media (Sigma). For GV oocyte isolation, ovaries were incubated in collagenase in M2 media ( $2 \mathrm{mg} / \mathrm{mL}$; Sigma) for $15 \mathrm{~min}$ at $37^{\circ} \mathrm{C}$ [51]. Follicles were collected and incubated in $0.05 \%$ trypsin/ EDTA in PBS (Sigma) for $15 \mathrm{~min}$ at $37^{\circ} \mathrm{C}$ to remove cumulus cells. Our group and others [51, 70] have demonstrated that acquisition of methylation marks at imprinted genes, including Snrpn, was completed by the late secondary follicle or early GV stages, by the time the oocyte is $60 \mu \mathrm{m}$ in diameter. For this reason, oocytes that were smaller than $60 \mu \mathrm{m}$ in diameter were excluded, with the majority of the oocytes analyzed falling between $70-80 \mu \mathrm{m}$ in diameter. To further prevent cumulus cell contamination, zonae pellucidae were removed from all oocytes by incubating the oocytes in 100\% acidic Tyrode's solution (Sigma) for $<1 \mathrm{~min}$ at room temperature.

For the superovulation only group, females received intraperitoneal (IP) injections of $10 \mathrm{IU}$ equine chorionic gonadotropin (eCG; Sigma) followed by $10 \mathrm{IU}$ human chorionic gonadotropin (hCG; Sigma) $46 \mathrm{~h}$ later [40]. Blastocysts were collected as described above. Superovulated MII oocytes were collected from the oviducts $18 \mathrm{~h}$ after the hCG injection and treated with $0.3 \mathrm{mg} / \mathrm{ml}$ 
hyaluronidase in M2 medium ( $0.3 \mathrm{mg} / \mathrm{mL}$; Sigma) to remove cumulus cells. Zonae pellucidae were removed from all MII oocytes as described above.

For the embryo culture only group, two-cell embryos were collected from the oviducts at E1.5 in M2 medium and washed in Whitten's medium [71]. Embryos were cultured for 3 days in Whitten's medium covered in mineral oil at $5 \% \mathrm{CO}_{2}, 5 \% \mathrm{O}_{2}$, and $37^{\circ} \mathrm{C}$ until the blastocyst stage.

For the superovulation and embryo culture group, females were superovulated as above, mated with B6 males, and two-cell embryos were collected on E1.5 and cultured for 3 days in Whitten's medium [71] covered in mineral oil at either $5 \% \mathrm{CO}_{2}, 5 \% \mathrm{O}_{2}$, and $90 \% \mathrm{~N}_{2}$, or $5 \%$ $\mathrm{CO}_{2}$ in air $\left(21 \% \mathrm{O}_{2}\right)$ at $37^{\circ} \mathrm{C}$ until the blastocyst stage. Note that in these experiments, we used 10 IU hormone dosages and/or embryo culture in Whitten's medium as the most tractable system for investigating imprinted methylation errors $[39,40]$.

\section{Imprinted DNA methylation analysis of individual oocytes and blastocysts}

All samples were processed immediately after collection without freezing. Individual oocytes or embryos were embedded into $10 \mu \mathrm{l}$ 2:1 agarose to lysis solution beads $(20 \mu \mathrm{L} \mathrm{3 \%}$ low melting point agarose; $8 \mu \mathrm{L}$ lysis buffer [100 mM Tris- $\mathrm{HCl}$, pH 7.5 (Bioshop), $500 \mathrm{mM} \mathrm{LiCl}$ (Sigma), $10 \mathrm{mM}$ ethylenediaminetetraacetic acid, pH 8.0 (Sigma), 1\% LiDS (Bioshop), and $5 \mathrm{mM}$ 1,4-dithiothreitol (Sigma)], $1 \mu \mathrm{l} 2 \mathrm{mg} / \mathrm{mL}$ proteinase $\mathrm{K}$ (Sigma), and $1 \mu \mathrm{l}$ 0.05\% Igepal (Sigma) under mineral oil [72]. A negative control (agarose bead without oocyte or embryo) was processed alongside for every eight samples. Bisulfite mutagenesis, nested PCR, and clonal sequencing were performed as described previously [72]. Briefly, samples in agarose beads were incubated in SDS lysis buffer (1\% sodium dodecyl sulfate in Tris-EDTA) at $50{ }^{\circ} \mathrm{C}$ overnight. The following day, the lysis buffer was replaced with mineral oil and samples were incubated at $90^{\circ} \mathrm{C}$ for 2.5 min to inactive the proteinase $\mathrm{K}$, then placed on ice for $10 \mathrm{~min}$. The DNA was denatured by incubation in $0.1 \mathrm{M}$ sodium hydroxide at $37^{\circ} \mathrm{C}$ for $15 \mathrm{~min}$. Samples were incubated in a $2.5 \mathrm{M}$ sodium bisulfite solution (3.8 g sodium bisulfite, $1 \mathrm{~mL} 0.125 \mathrm{M}$ hydroquinone, and 1 $\mathrm{mL} 3 \mathrm{M}$ sodium hydroxide in $5.5 \mathrm{~mL}$ water) under mineral oil at $50^{\circ} \mathrm{C}$ for $3-3.5 \mathrm{~h}$ depending on target gene (Additional file 1: Table S3). Samples were desulfonated with $0.3 \mathrm{M}$ sodium hydroxide at $37^{\circ} \mathrm{C}$ for $15 \mathrm{~min}$, and then were washed twice in Tris-EDTA and twice in water for $6 \mathrm{~min}$ each with shaking. Following sodium bisulfite treatment, DNA was amplified via two rounds of nested PCR using primers previous described for Snrpn, Kcnq1ot1, or H19 [40] (Additional file 1: Table S3). Agarose beads were added to the first round of PCR reactions containing Hot Start Ready-to-Go PCR beads (GE Healthcare), containing $0.2 \mu \mathrm{M}$ final concentration of outer primers and $9.6 \mathrm{ng} / \mathrm{ml}$ final concentration of tRNA as a carrier in the first-round reaction with mineral oil overlay. For the second-round reaction, $5 \mu \mathrm{l}$ of first round was added to a second $25 \mu$ ready-to-go PCR bead containing $0.2 \mu \mathrm{M}$ final concentration of the inner primers with mineral oil overlay. See Additional file 1: Table S3 for primers. Following successful amplification, second round PCR products were ligated with the pGEMT-EASY DNA ligation kit (Promega) overnight at $4{ }^{\circ} \mathrm{C}$. Ligations were transformed into competent $\mathrm{DH} 5 \alpha$ Escherichia coli cells (Zymo Research). Cloning success was determined with blue/white selection on LB/ampicillin/isopropyl $\beta$-D-1-thiogalactopyranoside/ $x$-galactose agar plates. For clonal sequencing, individual bacterial colonies were subjected to colony PCR with M13 primers (forward 5' to 3' CGCCAGGGTTTTCCCAGT CACGAC; reverse $5^{\prime}$ to $3^{\prime}$ TCACACAGGAAACAGC TATGAC). PCR amplicons of the correct size for each gene were verified by gel electrophoresis and were sent to Bio-Basic Inc. (Markham, QC, Canada) for sequencing. For oocytes, 5-8 clones were sequenced. Oocytes were excluded if the clones had more than one methylation pattern, suggestive of cumulus cell contamination. For embryos, 25-50 clones were sequenced from each sample for a final target of at least 7 independent clones each of maternal and paternal origin. Sequences were excluded for clones with less than $85 \%$ conversion; CAST7 and B6 polymorphisms in the same sequence, indicative of crossover; and identical number and location of unconverted CpG and non-CpG associated cytosines. Embryos were excluded if clones were of only maternal or paternal origin, indicating biased amplification. The methylation percentages for all unique maternal or paternal allele clones in an embryo were averaged to obtain the average methylation on the maternal or paternal alleles for the embryo.

\section{Statistical analysis}

Fisher's exact test (https://www.langsrud.com/fisher.htm) was used to calculate the significance of non-random association between groups of embryos. For the methylated maternal Snrpn, maternal Kcnq1ot1, or paternal H19 allele, embryos with $>75 \%$ methylation were designated as maintaining methylation and embryos with $\leq 75 \%$ methylation were designated as losing methylation. For the unmethylated paternal Snrpn, paternal Kcnq1ot1, or maternal $H 19$ allele, embryos with $\leq 20 \%$ methylation maintained methylation and embryos with $>20 \%$ methylation gained methylation. A one-sided, right-tailed test was used, as methylation changes were expected to be only in 
one direction (increase on the unmethylated allele or decrease on the methylated allele). $p$ values were considered significant at $p<0.05$.

\section{Additional file}

\begin{abstract}
Additional file 1: Figure S1. Correlation of mouse and human ages on which we based our mouse maternal age model. Figure S2. Imprinted methylation acquisition at the normally methylated maternal Snrpn ICR in individual mouse blastocysts from mothers of increasing age. Embryos from spontaneously ovulated (A) virgin (1.5 to 2 months old), (B) young maternal age ( $>2-6$ months old), (C) middle maternal age ( $>6-10$ months old), and (D) advanced maternal age $(>10$ months old) females ( $n=10-22$ embryos; $n=3-7$ females per age group). Each box encloses embryos analyzed from one female. Each block represents an individual embryo. Each line denotes an individual strand of DNA with the maternal allele [C57BL/6(CAST7)] on the left and the paternal allele (C57BL/6) on the right. Embryo designation is at the top left of each block and percent methylation is at the top right. Black circles, methylated CpGs; white circles, unmethylated CpGs; ARTs, assisted
\end{abstract} reproductive technologies. Figure S3. Imprinted methylation acquisition at the normally methylated maternal Kcnq1ot 1 ICR in individual blastocysts from mothers of increasing age. Embryos from spontaneously ovulated (A) virgin (1.5 to 2 months old), (B) young maternal age ( $>2-6$ months old), (C) middle maternal age ( $>6-10$ months old), and (D) advanced maternal age ( $>10$ months old) females ( $\mathrm{n}=9-16$ embryos; $n=3-5$ females per age group). See Figure $\mathbf{S} 2$ for details. Figure S4. Imprinted methylation acquisition at the normally methylated paternal H19 ICR in individual blastocysts from mothers of increasing age. Embryos from spontaneously ovulated (A) virgin (1.5 to 2 months old), (B) young maternal age ( $>2-6$ months old), (C) middle maternal age ( $>6-10$ months old), and (D) advanced maternal age $(>10$ months old) females ( $n=11-21$ embryos; $n=3-6$ females per age group). See Figure S2 for details. Figure S5. Imprinted methylation maintenance unaltered by maternal weight. (A) Positive correlation between maternal age and maternal weight. Black circles indicate female mice from which both oocytes and blastocysts were collected; white circles indicate female mice from which only oocytes were collected. (BD) No association between maternal weight and imprinted methylation at the (B) Snrpn, (C) Kcnq1ot1, or (D) H19 ICRs. Diamonds represent the mean methylation of maternal (red) or paternal (blue) alleles for individual embryos. Figure S6. Imprinted methylation maintenance at the normally methylated maternal Snrpn ICR in individual blastocysts from virgin females after ART treatment. (A) SO only; (B) $\mathrm{EC}$ only with $5 \% \mathrm{O}_{2} ;$ (C) SO+EC with $5 \% \mathrm{O}_{2} ;$ (D) SO+EC with $21 \% \mathrm{O}_{2}$ (n= 10-13 embryos; $n=3$ females per treatment group). Each box encloses embryos analyzed from one female. Each block denotes an individual embryo. Each line denotes an individual strand of DNA with the maternal allele [C57BL/6(CAST7)] on the left and the paternal allele (C57BL/6) on the right. Embryo designation is at the top left of each block and percent methylation is at the top right. Black circles indicate methylated CpGs, white circles indicate unmethylated CpGs. SO, superovulation; EC, in vitro embryo culture. Figure S7. Imprinted methylation maintenance at the normally methylated maternal Kcnq1ot1 ICR in individual blastocysts from virgin females after ART treatment. (A) $\mathrm{SO}$ only; (B) EC only with $5 \% \mathrm{O}_{2}$; (C) SO+EC with $5 \% \mathrm{O}^{2}$; (D) SO+EC with $21 \% \mathrm{O}_{2}$ ( $\mathrm{n}=$ 10-17 embryos; $n=3-4$ females per treatment group). See Figure $S 6$ for details. Figure S8. Imprinted methylation maintenance at the normally methylated paternal $H 19$ ICR in individual blastocysts from virgin females after ART treatment. (A) SO only; (B) EC only with $5 \% \mathrm{O}_{2 i}$ (C) SO+EC with $5 \% \mathrm{O}_{2}$ (D) SO+EC with $21 \% \mathrm{O}_{2}$ ( $\mathrm{n}=9-16$ embryos; $\mathrm{n}=3$ females per treatment group). See Figure $\mathbf{S 6}$ for details. Figure $\mathbf{S 9}$. At least one blastocyst from every ART-treated litter lost imprinted methylation on the normally methylated (A) Snrpn, (B) Kcnq1ot1, or (C) H19 ICRs. Diamonds represent the average percent methylation of maternal (red) or paternal (blue) alleles for individual embryos. ARTs, assisted reproductive technologies; SO and S, superovulation; EC and C, embryo culture; SC, superovulation plus embryo culture. Figure S10. Imprinted methylation maintenance at the normally methylated maternal Snrpn ICR in individual blastocysts from advanced maternal age females after ART treatment. Each box encloses embryos analyzed from one female. Each block denotes an individual embryo. Each line represents an individual strand of DNA with the maternal allele [C57BL/6(CAST7)] on the left and the paternal allele (C57BL/6) on the right. Embryo designation is at the top left of each block and percent methylation is at the top right. Black circles, methylated CpGs; white circles, unmethylated CpGs; SC, superovulation plus embryo culture. Figure S11. Imprinted methylation maintenance at the normally methylated maternal Kcnq1ot 1 ICR in individual blastocysts from advanced maternal age females after ART treatment. See Figure S10 for details. Figure S12. Imprinted methylation maintenance at the normally methylated paternal $\mathrm{H} 19$ ICR in individual blastocysts from advanced maternal age females after ART treatment. See Figure S10 for details. Table S1. Comparison of treatment groups using Fisher exact test. Table S2. Comparison of maternal age and treatment groups using Fisher exact test. Table S3. Bisulfite mutagenesis and PCR amplification.

\section{Abbreviations}

ART: Assisted reproductive technology; AS: Angelman syndrome; BWS: Beckwith-Wiedemann syndrome; DNMT: DNA methyltransferase; EC: Embryo culture; eCG: Equine chorionic gonadotropin; GV: Germinal vesicle; hCG: Human chorionic gonadotropin; ICR: Imprinting control region; MII: Meiosis II; PWS: Prader-Willi syndrome; SO: Superovulation; SRS: SilverRussell syndrome

\section{Acknowledgements}

The authors thank Carlee White for her technical expertise on bisulfite mutagenesis on oocytes and embryos.

\section{Authors' contributions}

AJK performed experiments, analyzed data, and wrote manuscript. MAC and CAP performed bisulfite mutagenesis and clonal sequencing. MPE performed clonal sequencing and helped with figure preparation. MRWM designed and supervised the project and helped with data interpretation and manuscript preparation. All authors have read and approved the final manuscript.

\section{Funding}

This work was supported by PA DOH 2018 Health Research Formula Fund, Magee-Womens Research Institute and the University of Pittsburgh to MRWM, and the Howard Hughes Medical Institute Medical Fellows Research Program to AJK.

\section{Availability of data and materials}

The datasets supporting the conclusions of this article are included within the article and its additional file.

\section{Ethics approval and consent to participate}

All experiments utilizing animals were approved by the Institutional Animal Care and Use Committee of the University of Pittsburgh (which is also the IACUC of record for Magee-Womens Research Institute, assurance no. A365401) and were performed in accordance with the NIH Guide for the Care and Use of Laboratory Animals.

\section{Consent for publication}

Not applicable

\section{Competing interests}

These authors declare that they have no competing interests. 
Received: 1 May 2019 Accepted: 23 September 2019 Published online: 26 November 2019

\section{References}

1. Ely DM, Hamilton BE. Trends in fertility and mother's age at first birth among rural and metropolitan counties: United States, 2007-2017. NCHS Data Brief. 2018:(323):1-8.

2. Sauer MV. Reproduction at an advanced maternal age and maternal health. Fertil Steril. 2015;103(5):1136-43.

3. Centers for Disease Control and Prevention ASfRM, Society for Assisted Reproductive Technology. : 2016 Assisted Reproductive Technology National Summary Report 2018.

4. Bushnik T, Cook JL, Yuzpe AA, Tough S, Collins J. Estimating the prevalence of infertility in Canada. Hum Reprod. 2012;27(3):738-46.

5. Chandra A, Copen CE, Stephen EH. Infertility and impaired fecundity in the United States, 1982-2010: data from the National Survey of Family Growth. Natl Health Stat Report. 2013;(67):1-18 11 p following 19

6. Kenny LC, Lavender T, McNamee R, O'Neill SM, Mills T, Khashan AS. Advanced maternal age and adverse pregnancy outcome: evidence from a large contemporary cohort. PLoS One. 2013;8(2):e56583.

7. Wu Y, Chen Y, Shen M, Guo Y, Wen SW, Lanes A, White RR, Adanlawo A, Walker M, Hua X. Adverse maternal and neonatal outcomes among singleton pregnancies in women of very advanced maternal age: a retrospective cohort study. BMC Pregnancy Childbirth. 2019;19(1):3.

8. Fraga MF, Ballestar E, Paz MF, Ropero S, Setien F, Ballestar ML, Heine-Suner D, Cigudosa JC, Urioste M, Benitez J, et al. Epigenetic differences arise during the lifetime of monozygotic twins. Proc Natl Acad Sci U S A. 2005; 102(30):10604-9.

9. Maegawa S, Hinkal G, Kim HS, Shen L, Zhang L, Zhang J, Zhang N, Liang S, Donehower LA, Issa JP. Widespread and tissue specific age-related DNA methylation changes in mice. Genome Res. 2010;20(3):332-40.

10. Christiansen L, Lenart A, Tan Q, Vaupel JW, Aviv A, McGue M, Christensen K. DNA methylation age is associated with mortality in a longitudinal Danish twin study. Aging Cell. 2016;15(1):149-54.

11. Tan Q, Heijmans BT, Hjelmborg JV, Soerensen M, Christensen K, Christiansen L. Epigenetic drift in the aging genome: a ten-year follow-up in an elderly twin cohort. Int J Epidemiol. 2016:45(4):1146-58.

12. Richardson SJ, Senikas V, Nelson JF. Follicular depletion during the menopausal transition: evidence for accelerated loss and ultimate exhaustion. J Clin Endocrinol Metab. 1987;65(6):1231-7.

13. Wallace $\mathrm{WH}$, Kelsey TW. Human ovarian reserve from conception to the menopause. PLoS One. 2010;5(1):e8772.

14. Yue MX, Fu XW, Zhou GB, Hou YP, Du M, Wang L, Zhu SE. Abnormal DNA methylation in oocytes could be associated with a decrease in reproductive potential in old mice. J Assist Reprod Genet. 2012;29(7):643-50.

15. Hamatani T, Falco G, Carter MG, Akutsu H, Stagg CA, Sharov AA, Dudekula $D B$, VanBuren $V$, Ko MS. Age-associated alteration of gene expression patterns in mouse oocytes. Hum Mol Genet. 2004;13(19):2263-78.

16. Barlow DP, Bartolomei MS. Genomic Imprinting in Mammals. In: Allis CD, Jenuwein T, Rinberg D, Caparros M-L, editors. Epigenetics. Cold Spring Harbor: Cold Spring Harbor Laboratory Press; 2007. p. 357-75.

17. Denomme MM, Mann MRW. Genomic imprints as a model for the analysis of epigenetic stability during assisted reproductive technologies. Reproduction. 2012;144(4):393-409.

18. MacDonald WA, MRW M. Epigenetic regulation of genomic imprinting from germ line to preimplantation. Mol Reprod Dev. 2014;81(2):126-40.

19. Cox GF, Burger J, Lip V, Mau UA, Sperling K, Wu BL, Horsthemke B. Intracytoplasmic sperm injection may increase the risk of imprinting defects. Am J Hum Genet. 2002;71(1):162-4.

20. Orstavik KH, Eiklid K, van der Hagen CB, Spetalen S, Kierulf K, Skjeldal O, Buiting K. Another case of imprinting defect in a girl with Angelman syndrome who was conceived by intracytoplasmic semen injection. Am J Hum Genet. 2003:72(1):218-9.

21. Maher ER, Brueton LA, Bowdin SC, Luharia A, Cooper W, Cole TR, Macdonald F, Sampson JR, Barratt CL, Reik W, et al. Beckwith-Wiedemann syndrome and assisted reproduction technology (ART). J Med Genet. 2003; 40(1):62-4.

22. DeBaun MR, Niemitz EL, Feinberg AP. Association of in vitro fertilization with Beckwith-Wiedemann syndrome and epigenetic alterations of LIT1 and H19. Am J Hum Genet. 2003;72(1):156-60.
23. Gicquel C, Gaston V, Mandelbaum J, Siffroi JP, Flahault A, Le Bouc Y. In vitro fertilization may increase the risk of Beckwith-Wiedemann syndrome related to the abnormal imprinting of the KCN1OT gene. Am J Hum Genet. 2003; 72(5):1338-41.

24. Halliday J, Oke K, Breheny S, Algar E. D JA: Beckwith-Wiedemann syndrome and IVF: a case-control study. Am J Hum Genet. 2004;75(3):526-8.

25. Lim D, Bowdin SC, Tee L, Kirby GA, Blair E, Fryer A, Lam W, Oley C, Cole T, Brueton LA, et al. Clinical and molecular genetic features of BeckwithWiedemann syndrome associated with assisted reproductive technologies. Hum Reprod. 2009;24(3):741-7.

26. Bliek J, Terhal P, van den Bogaard MJ, Maas S, Hamel B, Salieb-Beugelaar G, Simon M, Letteboer T, van der Smagt J, Kroes H, et al. Hypomethylation of the H19 gene causes not only Silver-Russell syndrome (SRS) but also isolated asymmetry or an SRS-like phenotype. Am J Hum Genet. 2006;78(4):604-14.

27. Hiura H, Okae H, Miyauchi N, Sato F, Sato A, Van De Pette M, John RM, Kagami M, Nakai K, Soejima H, et al. Characterization of DNA methylation errors in patients with imprinting disorders conceived by assisted reproduction technologies. Hum Reprod. 2012;27(8):2541-8.

28. Kagami M, Nagai T, Fukami M, Yamazawa K, Ogata T. Silver-Russell syndrome in a girl born after in vitro fertilization: partial hypermethylation at the differentially methylated region of PEG1/MEST. J Assist Reprod Genet. 2007:24(4):131-6.

29. Chopra M, Amor DJ, Sutton L, Algar E, Mowat D. Russell-Silver syndrome due to paternal H19/IGF2 hypomethylation in a patient conceived using intracytoplasmic sperm injection. Reprod Biomed Online. 2010;20(6):843-7.

30. Cocchi G, Marsico C, Cosentino A, Spadoni C, Rocca A, De Crescenzo A, Riccio A. Silver-Russell syndrome due to paternal H19/IGF2 hypomethylation in a twin girl born after in vitro fertilization. Am J Med Genet A. 2013; 161A(10):2652-25.

31. Vermeiden JP, Bernardus RE. Are imprinting disorders more prevalent after human in vitro fertilization or intracytoplasmic sperm injection? Fertil Steril. 2013;99(3):642-51

32. Chiba H, Hiura H, Okae H, Miyauchi N, Sato F, Sato A, Arima T. DNA methylation errors in imprinting disorders and assisted reproductive technology. Pediatr Int. 2013:55(5):542-9.

33. Ludwig M, Katalinic A, Gross S, Sutcliffe A, Varon R, Horsthemke B. Increased prevalence of imprinting defects in patients with Angelman syndrome born to subfertile couples. J Med Genet. 2005;42(4):289-91.

34. Cortessis VK, Azadian M, Buxbaum J, Sanogo F, Song AY, Sriprasert I, Wei PC, Yu J, Chung K, Siegmund KD. Comprehensive meta-analysis reveals association between multiple imprinting disorders and conception by assisted reproductive technology. J Assist Reprod Genet. 2018;35(6):943-52.

35. Hattori H, Hiura H, Kitamura A, Miyauchi N, Kobayashi N, Takahashi S, Okae $H$, Kyono K, Kagami M, Ogata T, et al. Association of four imprinting disorders and ART. Clin Epigenetics. 2019;11(1):21.

36. Doherty AS, Mann MRW, Tremblay KD, Bartolomei MS, Schultz RM. Differential effects of culture on imprinted $\mathrm{H} 19$ expression in the preimplantation mouse embryo. Biol Reprod. 2000:62:1526-35.

37. Mann MR, Lee SS, Doherty AS, Verona RI, Nolen LD, Schultz RM, Bartolomei MS. Selective loss of imprinting in the placenta following preimplantation development in culture. Development. 2004;131(15):3727-35.

38. Market Velker BA, Denomme MM, Mann MR. Loss of genomic imprinting in mouse embryos with fast rates of preimplantation development in culture. Biol Reprod. 2012:86(5):143 141-116.

39. Market-Velker BA, Fernandes AD, Mann MRW. Side-by-side comparison of five commercial media systems in a mouse model: suboptimal in vitro culture interferes with imprint maintenance. Biology of Reproduction. 2010; 83(6):938-50.

40. Market-Velker BA, Zhang L, Magri LS, Bonvissuto AC, Mann MRW. Dual effects of superovulation: loss of maternal and paternal imprinted methylation in a dose-dependent manner. Human Molecular Genetics. 2010;19(1):36-51.

41. Velker BAM, Denomme MM, Krafty RT, Mann MRW. Maintenance of Mest imprinted methylation in blastocyst-stage mouse embryos is less stable than other imprinted loci following superovulation or embryo culture. Environ Epigenet. 2017;3(3):dvx015.

42. White CR, Denomme MM, Tekpetey FR, Feyles V, Power SGA, Mann MRW. High frequency of imprinted methylation errors in human preimplantation embryos. Sci Rep. 2015:5:17311.

43. Denomme MM, Zhang L, Mann MR. Embryonic imprinting perturbations do not originate from superovulation-induced defects in DNA methylation acquisition. Fertil Steril. 2011;96(3):734-8 e732. 
44. Dutta S, Sengupta P. Men and mice: Relating their ages. Life Sci. 2016 152:244-8.

45. Ferando I, Faas GC, Mody I. Diminished KCC2 confounds synapse specificity of LTP during senescence. Nat Neurosci. 2016;19(9):1197-200.

46. White CR, MacDonald WA, MRW M. Conservation of DNA methylation programming between mouse and human gametes and preimplantation embryos. Biol Reprod. 2016;95(3):61.

47. Dumoulin JC, Vanvuchelen RC, Land JA, Pieters MH, Geraedts JP, Evers JL. Effect of oxygen concentration on in vitro fertilization and embryo culture in the human and the mouse. Fertil Steril. 1995;63(1):115-9.

48. Pabon JE Jr, Findley WE, Gibbons WE. The toxic effect of short exposures to the atmospheric oxygen concentration on early mouse embryonic development. Fertil Steril. 1989;51(5):896-900.

49. Rinaudo PF, Giritharan G, Talbi S, Dobson AT, Schultz RM. Effects of oxygen tension on gene expression in preimplantation mouse embryos. Fertil Steril. 2006;86(4 Suppl):1252-65.

50. Feil D, Lane M, Roberts CT, Kelley RL, Edwards LJ, Thompson JG, Kind KL. Effect of culturing mouse embryos under different oxygen concentrations on subsequent fetal and placental development. J Physiol. 2006;572(Pt 1):87-96.

51. Denomme MM, White CR, Gillio-Meina C, Macdonald WA, Deroo BJ, Kidder GM, Mann MR. Compromised fertility disrupts Peg1 but not Snrpn and Peg3 imprinted methylation acquisition in mouse oocytes. Front Genet. 2012;3:129.

52. Barzilai $N$, Huffman DM, Muzumdar RH, Bartke A. The critical role of metabolic pathways in aging. Diabetes. 2012;61(6):1315-22.

53. Dunford AR, Sangster JM. Maternal and paternal periconceptional nutrition as an indicator of offspring metabolic syndrome risk in later life through epigenetic imprinting: a systematic review. Diabetes Metab Syndr. 2017; 11(Suppl 2):S655-62.

54. Godfrey KM, Reynolds RM, Prescott SL, Nyirenda M, Jaddoe WW, Eriksson JG, Broekman BF. Influence of maternal obesity on the long-term health of offspring. Lancet Diabetes Endocrinol. 2017;5(1):53-64.

55. Agarwal P, Morriseau TS, Kereliuk SM, Doucette CA, Wicklow BA, Dolinsky WW. Maternal obesity, diabetes during pregnancy and epigenetic mechanisms that influence the developmental origins of cardiometabolic disease in the offspring. Crit Rev Clin Lab Sci. 2018;55(2):71-101.

56. de Waal E, Mak W, Calhoun S, Stein P, Ord T, Krapp C, Coutifaris C, Schultz $\mathrm{RM}$, Bartolomei MS. In vitro culture increases the frequency of stochastic epigenetic errors at imprinted genes in placental tissues from mouse concepti produced through assisted reproductive technologies. Biol Reprod. 2014:90(2):22.

57. Ghosh J, Coutifaris C, Sapienza C, Mainigi M. Global DNA methylation levels are altered by modifiable clinical manipulations in assisted reproductive technologies. Clin Epigenetics. 2017;9:14.

58. Lopes FL, Fortier AL, Darricarrere N, Chan D, Arnold DR, Trasler JM. Reproductive and epigenetic outcomes associated with aging mouse oocytes. Hum Mol Genet. 2009;18(11):2032-44

59. Paczkowski M, Schoolcraft WB, Krisher RL. Dysregulation of methylation and expression of imprinted genes in oocytes and reproductive tissues in mice of advanced maternal age. J Assist Reprod Genet. 2015;32(5):713-23.

60. Rivera RM, Stein P, Weaver JR, Mager J, Schultz RM, Bartolomei MS. Manipulations of mouse embryos prior to implantation result in aberrant expression of imprinted genes on day 9.5 of development. Hum Mol Genet. 2008;17(1):1-14

61. Turan N, Katari S, Gerson LF, Chalian R, Foster MW, Gaughan JP, Coutifaris C, Sapienza C. Inter- and intra-individual variation in allele-specific DNA methylation and gene expression in children conceived using assisted reproductive technology. PLoS Genet. 2010;6(7):e1001033.

62. de Waal E, Vrooman LA, Fischer E, Ord T, Mainigi MA, Coutifaris C, Schultz RM, Bartolomei MS. The cumulative effect of assisted reproduction procedures on placental development and epigenetic perturbations in a mouse model. Hum Mol Genet. 2015;24(24):6975-85.

63. Adkins RM, Thomas F, Tylavsky FA, Krushkal J. Parental ages and levels of DNA methylation in the newborn are correlated. BMC Med Genet. 2011;12:47.

64. Markunas CA, Wilcox AJ, Xu Z, Joubert BR, Harlid S, Panduri V, Haberg SE, Nystad W, London SJ, Sandler DP, et al. Maternal age at delivery is associated with an epigenetic signature in both newborns and adults. PLoS One. 2016;11(7):e0156361.

65. Moore AM, Xu Z, Kolli RT, White AJ, Sandler DP, Taylor JA. Persistent epigenetic changes in adult daughters of older mothers. Epigenetics. 2019; 14(5):467-76.
66. Akiyama T, Nagata M, Aoki F. Inadequate histone deacetylation during oocyte meiosis causes aneuploidy and embryo death in mice. Proc Nat Acad Sci U S A. 2006;103(19):7339-44.

67. van den Berg IM, Eleveld C, van der Hoeven M, Birnie E, Steegers EA, Galjaard RJ, Laven JS, van Doorninck JH. Defective deacetylation of histone $4 \mathrm{~K} 12$ in human oocytes is associated with advanced maternal age and chromosome misalignment. Hum Reprod. 2011;26(5):1181-90.

68. Manosalva I, Gonzalez A. Aging changes the chromatin configuration and histone methylation of mouse oocytes at germinal vesicle stage. Theriogenology. 2010;74(9):1539-47.

69. Mann M, Chung Y, Nolen L, Verona R, Latham K, Bartolomei M. Disruption of imprinted gene methylation and expression in cloned preimplantation stage mouse embryos. Biology of Reproduction. 2003;69(3):902-14.

70. Hiura H, Obata Y, Komiyama J, Shirai M, Kono T. Oocyte growth-dependent progression of maternal imprinting in mice. Genes Cells. 2006;11(4):353-61.

71. Whitten WK. Nutrient requirements for the culture of preimplantation mouse embryo in vitro. Adv Biosci. 1971;6:129-39.

72. Denomme MM, Zhang L, Mann MRW. Single oocyte bisulfite mutagenesis. J Vis Exp. 2012;(64)). https://doi.org/10.3791/4046.

\section{Publisher's Note}

Springer Nature remains neutral with regard to jurisdictional claims in published maps and institutional affiliations.
Ready to submit your research? Choose BMC and benefit from:

- fast, convenient online submission

- thorough peer review by experienced researchers in your field

- rapid publication on acceptance

- support for research data, including large and complex data types

- gold Open Access which fosters wider collaboration and increased citations

- maximum visibility for your research: over $100 \mathrm{M}$ website views per year

At BMC, research is always in progress.

Learn more biomedcentral.com/submissions 